Silva dos SANTOS, FaBIAne.

Artista visual, Doctora en Artes Visuales e Intermedia por la Universidad Politécnica de Valencia.

\title{
El arte participativo, acciones reivindicativas y colaborativas que buscan la cohesión social. De lo local a lo global.
}

\section{Participatory art, demanding and collaborative actions that seek social cohesion. From the local to the global.}

\author{
TIPO DE TRABAJO: \\ Comunicación. \\ PALABRAS CLAVE: \\ Feminismo, Espacio privado/público, Arte en Red , Arte participativo, Craftivismo.
}

KEY WORDS:

Feminism, Private / public space, Networking, Participatory art, Craftivism.

\section{RESUMEN.}

El creciente interés por el arte político, viene marcado por un cambio en las formas de visualizar, conceptuar, producir y visibilizar las intervenciones artísticas en el espacio público, que pasan por vincularse directamente con las nuevas formas del activismo político. Hablamos de la manera directa en que el arte se relaciona con el contexto, a partir de un proceso colaborativo donde el espectador tiene un papel fundamental en el proceso de desarrollo de la obra. Los años ochenta marcaron la búsqueda de la participación del público en proyectos de ocupación de espacio, en un proceso de experimentación de formas de socialización y ocupación. Nuevas estrategias para la producción de la obra artística, convierten la creación en una acción social e integran al espectador en el proceso, fomentando la integración de distintas personas que conviven en un contexto social terminado.

Trataremos de abordar algunas acciones de arte participativo en las que muchos creadores están actualmente desarrollando y que no serían posible sin la colaboración de diferentes personas y colectivos, proyectos como CraftCabanyal, Tejiendo La Calle, Mujeres y Punto, entre otros, analizando su proceso de construcción y desarrollo.

\section{ABSTRACT.}

The growing interest in political art is marked by a change in the ways of visualizing, conceptualizing, producing and making visible the artistic interventions in the public space, which are linked directly to the new forms of political activism. We speak of the direct way in which art relates to the context, based on a collaborative process where the viewer plays a fundamental role in the development process of the work. The eighties marked the search of the participation of the public in projects of occupation of space, in a process of experimentation of forms of socialization and occupation. New strategies for the production of the artistic work, convert the creation into a social action and integrate the viewer in the process, encouraging the integration of different people who live in a social context.

We will try to approach some participatory art actions in which many creators are currently developing and that would not be possible without the collaboration of different people and groups, projects like CraftCabanyal, Tejiendo La Calle, Mujeres y Punto, among others, analyzing their construction process and development. 


\section{CONTENIDO}

Yo definiría el arte público como cualquier tipo de obra de libre acceso que se preocupa, desafía, implica, y tiene en cuenta la opinión del público para quien o con quien ha sido realizada, respetando la comunidad y al medio. ${ }^{1}$

Nuevas estrategias surgen para encajar las prácticas artísticas en el contexto social, buscando la participación del público, el dialogo con el entorno desde el público/privado. Pero podemos retroceder en el tiempo y recuperar los primeros happenings protagonizados por Allan Kaprow, donde invitaba al espectador físicamente e intelectualmente a interactuar con el arte y formar parte de la obra. Surge el arte que permite o instiga al visitante a manipular, incorporar, expresarse, convirtiéndose en verdaderos objetos o sujetos de la obra. Obras como la de Félix Gonzales-Torres - Montaña de caramelos envueltos (Sin título,1991) que invita al espectador a coger y consumir los caramelos, o la acción de Yoko Ono "Cut piece" (Corte un pedazo) - 1964, donde los espectadores fueron invitados a cortar con tijeras un trozo de su ropa, son acciones que propician una obra donde la reciprocidad y la participación proactiva se hace presente.

Nuevas formas aparecen en el cambio de paradigma estético y cultural, el arte pasa a tener una función social, el espectador pasa a tener un protagonismo, cobrando un papel fundamental en la construcción de la obra, donde el espacio público se converte en el lugar intervenido relacionándose con el arte y su contexto local. "A lo largo de los siglos XIX y XX, el artista va ganando un importante rol como agente del cambio social a partir de las discusiones y acciones vanguardistas en torno al contenido político del arte. Al abandonar este los museos y espacios consagrados de la cultura burguesa, se convierte en parte de la vida popular debido a su relación con el espacio y sus problemáticas. A partir de los años ochenta se acentúa la relación entre arte y política, multiplicándose las iniciativas artísticas mediante las cuales los artistas buscaban promover la participación de personas del público en proyectos tales como experimentación de formas de socialización y ocupación de espacios locales, etc." ${ }^{2}$

Al largo de los años setenta surge dentro del arte contemporáneo intervenciones artísticas comprometidas e implicadas dentro del contexto social, artistas como Hans Haaker, Marta Rosler, Camilio Vergara, Krzysztof Wodiczko, entre otros, expresaban en sus obras ese compromiso con el contexto. Esta vertiente de arte fue acogida por distintos movimientos sociales, como el ecologismo, el feminismo y otros. Siguiendo la línea del arte político surge el arte colaborativo, donde las propuestas artísticas se desarrollan dentro de colectivos sociales con ciertas problemáticas. El arte busca visibilizar e instigar que las ciudadanías se involucren en una tentativa de resolución del conflicto, así como en la integración o reforzando la autoestima de sus integrantes. "El arte colaborativo, por lo tanto, busca que se revise el papel del artista, del público y de la obra, cuestionando la concepción tradicional del arte. El artista se coloca en situación de igualdad con el espectador, y el espectador deja de ser el receptor pasivo y pasa a integrarse activamente en el proceso de desarrollo de la obra. La obra recibe también una nueva percepción, que surge en relación a su contexto, y el proceso gana trascendencia a partir del conjunto de sus actores y de sus acciones: la obra resultante deja de ser una finalidad y se convierte en una evidencia directa que presenta un resultado proveniente de la multiplicidad de acciones creativas individuales. En ese proceso de desarrollo de la propuesta, para que su finalidad sea alcanzada, es importante que participen todos en la elaboración del proyecto; así se genera una metodología para que, en general, todos los participantes se relacionen entre sí, fomentando la integración de diferentes grupos que conviven en un determinado contexto social. ${ }^{3}$

Actualmente varios artistas vienen desarrollando acciones colaborativas con diferentes colectivos en una acción de Craftvismo, en un cruzamiento de lo artesanal con el activismo. Las prácticas artesanales relacionadas directamente con el espacio privado pasan a tener visibilidad en el espacio público, dando visibilidad a una situación, o un reclamo social en esa sociedad que durante mucho tiempo ha cultivado un contexto social individualista. La transformación social que actualmente venimos experimentando, en un cambio de paradigma de las relaciones sociales y políticas donde la participación, la colaboración y la colectividad vienen siendo las grandes acciones para lograr el cambio del desarrollo social. Y una de las herramientas que actualmente vienen sirviendo como vehículo para conquistar esta cohesión social es el arte, que promueve un trabajo creativo, generando un espacio de resistencia, transformando o visibilizando una problemática.

Trataremos aquí de presentar algunas iniciativas que vienen siendo llevando a cabo por diferentes colectivos a partir de una propuesta artística para la realización de una obra colaborativa, proyectos como CraftCabanyal, Tejiendo La Calle, Mujeres y Punto, entre otros, dialogará con otras propuestas que están siendo desarrolladas en otros lugares con diferentes interlocutores. Estas iniciativas utilizan la Red como herramienta complementaria a sus acciones, para difundir, generando conexiones, a través de su blog, páginas webs y de

\footnotetext{
${ }^{1}$ Lippard, Lucy R. (2001). Mirando alrededor: dónde estamos y dónde podríamos estar. Modos de hacer. Arte crítico, esfera pública y ${ }^{2}$ Silva Dos Santos, FC. (2015). Mujer, casa y nuevos medios: el arte de tejer en red, una propuesta experimental artística neomedial [Tesis doctoral no publicada]. Universitat Politècnica de València. doi:10.4995/Thesis/10251/58774, pag.444, In: http://hdl.handle.net/10251/58774

${ }^{3}$ ídem: pag. 446 .
} 
las redes sociales, donde la integración y el intercambio de conocimientos amplia la posibilidad de conexiones, propiciando una experiencia que rompe las fronteras espacio- temporales.

Mujeres y punto es un colectivo de mujeres que surge en 2012 en el municipio de Aldaia, donde se reúnen una vez a la semana para tejer. Inicialmente tenía el propósito de reunirse para aprender o reaprender el acto de tejer, realizan su primera intervención en las calles de Aldaia con intervenciones en las paredes del pueblo. Con el pasar del tiempo el grupo participa de diferentes iniciativas de carácter artístico-cultural, trazando objetivos para desarrollar acciones anuales en diferentes contextos, las cuales hacen con que las aportaciones de ese colectivo pasen a ver visibilizadas, compartido experiencias generando cohesión social. A partir de diversas aportaciones en 2013 realizaron la intervención pública en el Festival de arte público, "XVI Mostra d'art públic per a joves creadors" (Universitat de València, donde a través de una gran pancarta hecha en ganchillo lazaron al espacio público un mensaje de optimismo, donde se podía leer : "No aparques tus sueños".

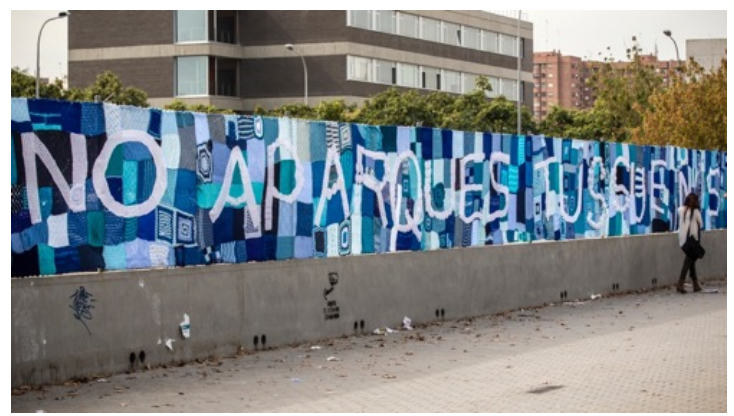

Mujeres y Punto. Foto: Miguel Lorenzo. http://www.makma.net/tag/profesora-de-la-universitat-devalencia/

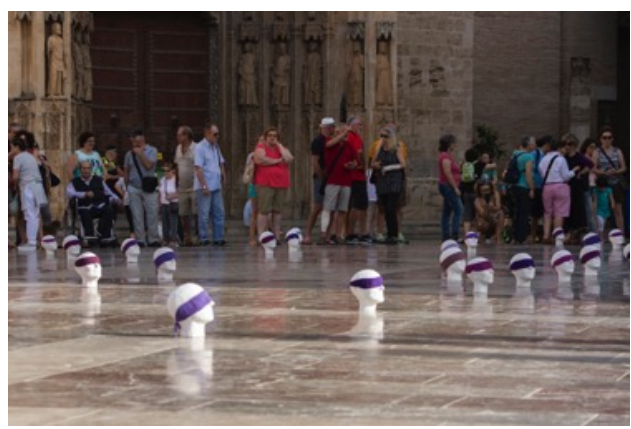

00 cabezas, 100 mujeres, 100 vendas moradas. Foto: Rosi Moreno. http://uanflores.blogspot.com.es/

Entre otras intervenciones también destacamos el trabajo realizado junto al escultor Juan Flores a partir de la acción que reproducía diferentes cabezas realizadas a través de molde de escayola con la colaboración de diferentes colectivos como Ciutat Vella Batega, Salvem el Cabanyal y Horta és Futur en una intervención de 100 cabezas en la plaza del Ayuntamiento de Valencia. En 2015 se une al proyecto de Mujeres y punto "No quiero ser huevo frito" el cual remite a la teoría del aislamiento de la mujer sometida al hombre. A partir de ahí realizan una campaña poniendo en relieve la poca presencia femenina en las elecciones durante las elecciones de junio de 2016. Varias cabezas de mujeres pasan a formar parte de esta intervención con una mordaza en punto color morado realizadas por Mujeres y punto que tapaba la boca de cada cabeza.

Las intervenciones realizadas por Mujeres y punto se enmarcan dentro de la vertiente del Craftivismo que se denomina "urban knitting" (Tejido urbano) o Guerrilla Knitting, o punto de graffiti arte urbano que decora el espacio público con labores de punto y ganchillo. Un movimiento que tiene origen en 2005, cuando en la ciudad de Houston los monumentos aparece decorados con labores de punto y ganchillo, pasando a ganar adeptos en todo el mundo, y en España en la ciudades de Barcelona, Bilbao y Valencia ha generado una red en vuelta de Urban Knitting, llegando el fenómeno ha diversas ciudades del norte al sur del país. El acto de reunirse una vez a la semana y realizar una acción en pro de una causa plasmando en el espacio público, reivindicando el bello y principalmente el acto del hacer manual (Hand-Made), donde el concepto de reciclaje, socialización, igualdad hace parte de sus principios. 


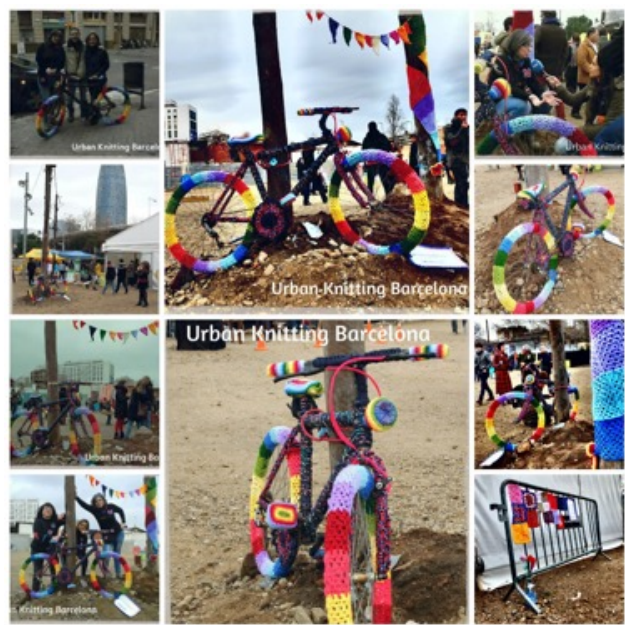

\section{Urban Knitting Barcelona}

http://urbanknittingbarcelona.blogspot.com.es/

En 2013 surge el proyecto participativo Tejiendo Calle coordinado por Marina Fernández, realizado en las calles de Valverde de La Vera (Cáceres), que consiste el realizar parasoles con la técnica de ganchillo XXL, instalados en el mes de agosto, utilizando materiales reciclados como bolsas de compras convirtiéndolas en largas tiras que pasan a ser el hilo para tejer. Realizado en colaboración con el Asociación Cultural y Juvenil La Chorrera, juntamente con la comunidad del pueblo donde una vez al mes de se reúnen para tejer a través de talleres de producción en la plaza del pueblo donde se comparten experiencias y conocimientos. Un proyecto intergeneracional, en el que la cohesión social es su principal elemento y que utiliza las Red como para divulgar las convocatorias de cada año
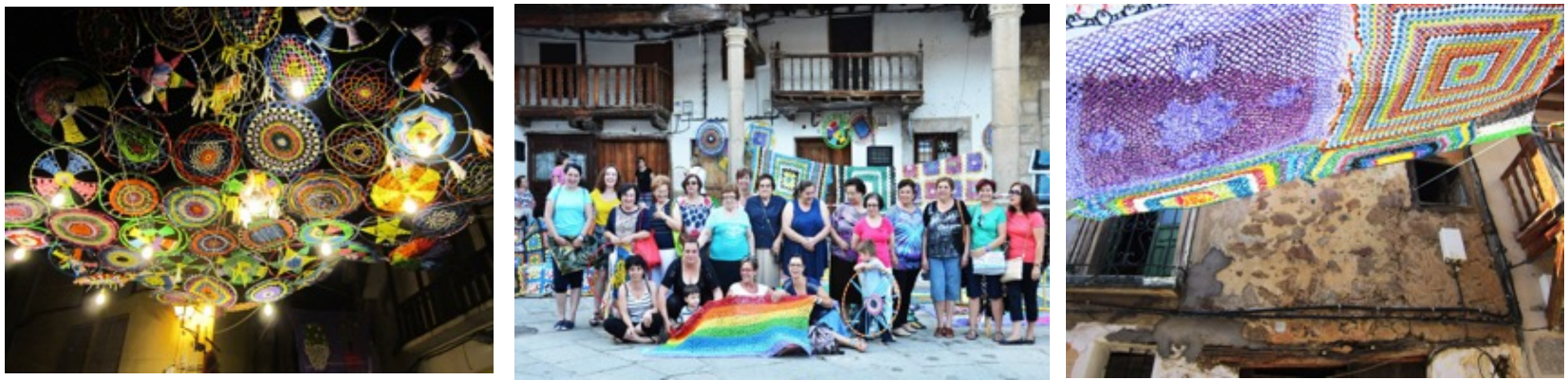

Tejiendo Calle - https://submarina.info/tejiendo-la-calle/

En contra punto, pero con similitudes al proyecto Tejiendo Calle, se encuentra el proyecto Crochet Coral, iniciado en 2015 en Los Ángeles por Margaret Wertheim y Christine Wertheim para el The Institute For Figuring, que consiste en realizar a través del ganchillo, mezclando hilo con la basura de plástico, arrecifes en lana, generando ambientes marinos recreados a partir de la intersección del arte de tejer, con las matemáticas, con la ciencia, como signo de protesta contra el calentamiento global. Inspirados en la técnica de "Crochet hiperbólico", "flecos", anémonas almenadas "babosas de mar", y "corales" modelados con estos métodos, que van creciendo y formando sub-arrecifes a través de colaboraciones de comunidades que están por todo el mundo desde Chicago, Nueva York y Londres, Melbourne, Dublín y Ciudad del Cabo. El proyecto sigue abierto a colaboraciones para realización colectiva de arrecifes para el instituto, a través de su página web (http://crochetcoralreef.org) se puede registrar y encontrar todas informaciones como derecho de autor, tamaño, duración del proyecto, proporcionando un apoyo a diferentes etapas del proceso. El proyecto viene siendo presentado a través de exposiciones, charlas, en diferentes partes del mundo. Es un proyecto colaborativo que crece con la colaboración proliferándose como los arrecifes vivos que envían semillas de regeneración. 


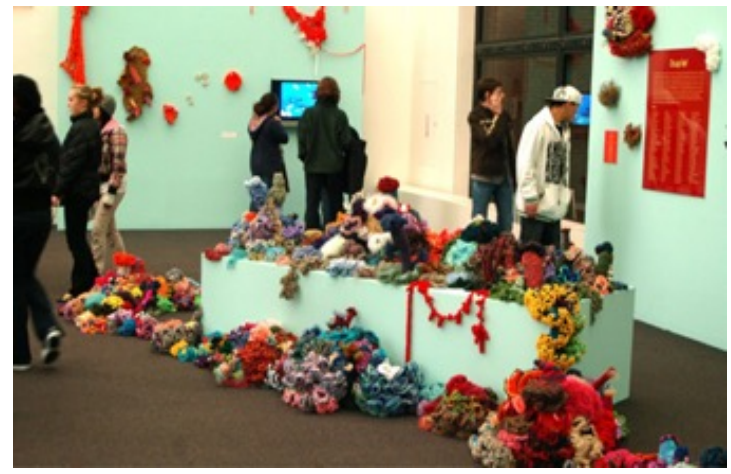

El arrecife de Chicago muestra en el Centro Cultural de Chicago (otoño de 2007). El arrecife de Chicago fue encabezado por el Jane Addams Hull-House Museum, en conjunción con el Festival de Humanidades de Chicago.

In: http://crochetcoralreef.org/satellite/chicago.php

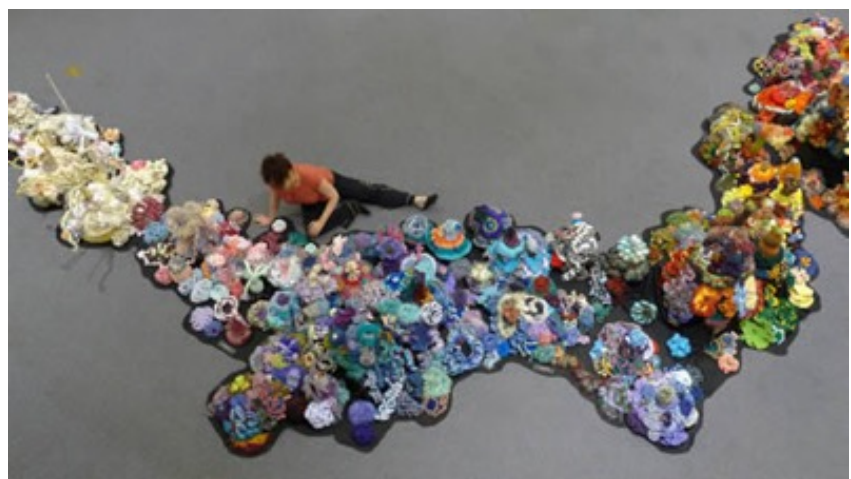

Christine Wertheim curador de Coral Popular (corales de Nueva York y Chicago foto), ya que se instaló en Scottsdale, AZ. Foto por la FIB.

In: http://crochetcoralreef.org/satellite/index.php

En 2015 la artista Cornelia Parker ha realizado la obra Magna Carta (un bordado), un bordado de la representación completa del texto y imágenes de la Carta Magna como aparece en la página de la enciclopedia online Wikipedia en 2014. Un bordado en gran formado en una tela de 1,5 metros de ancho y casi 40 metros de largo, dividida en 87 partes las cuales fueron enviadas a 200 personas para colaborar en el bordado de la obra, entre ellas presos, defensores de los derechos civiles, parlamentarios, abogados, barones y artistas. La mayor parte del trabajo fue realizado por 36 presos de 13 cárceles diferentes en Inglaterra. También ha participado del proyecto personalidades como Edward Snowden bordó la palabra 'libertad' y Jimmy Wales el creador de Wikipedia que bordó la palabra Wikipedia, entre otros. Esta obra hace parte del homenaje a los 800 aniversario de la Carta Magna, la cual estuvo en exposición en el vestíbulo de la biblioteca británica.

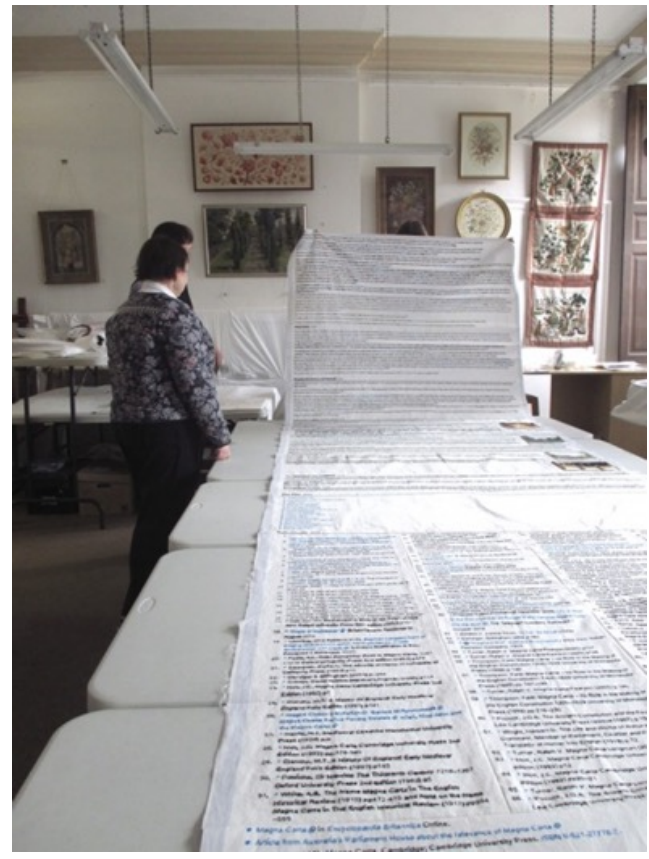

Cornelia Parker - Carta Magna

In: http://www.royalneedlework.org.uk/galleries/images/35/

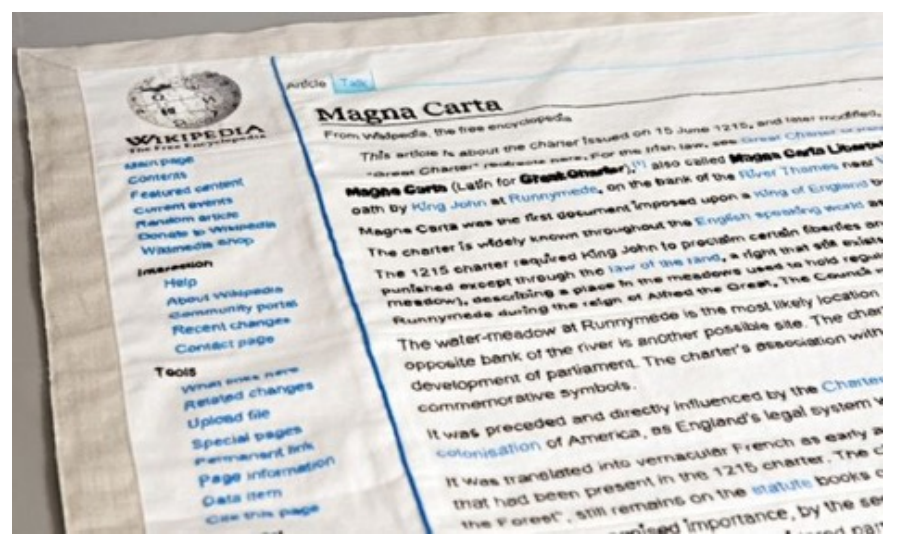

Cornelia Parker - Carta Magna In:https://www.theguardian.com/artanddesign/jonathanjonesblog/2015/may/1 4/magna-carta-an-embroidery-cornelia-parker-british-library-wikipediaprisoners-jarvis-cocker 
Desde 2013 realizamos un proyecto CraftCabanyal ${ }^{4}$, propuesto a la Plataforma Salvem el Cabanyal ${ }^{5}$, dónde venimos realizando diversas intervenciones con propuestas de arte colaborativo en los que los vecinos se convierten en artífices de sus propias intervenciones. La última propuesta que se está llevando a cabo, iniciada en 2015 teniendo como referencia la obra de Cornelia Parker, es el bordado de la “Orden Ministerial CUL/3631/2009 de 29 de diciembre para su cumplimiento" ${ }^{\prime 6}$. A través de una acción artística, utilizamos la Red para convocar a los vecinos y vecinas, simpatizantes para bordar entre todos, las 17 páginas de la orden ministerial en tela $(1,5 \times 2 \mathrm{~m}$. cada página), que ha amparado y salvaguardado el patrimonio del barrio Cabanyal - Canyamelar - Cap de França. Es un homenaje a la Orden Ministerial y al mismo tiempo una acción de arte participativo / reivindicativa para su cumplimiento. Es un proyecto que está en proceso, donde ya ha colaborado más de 380 personas hasta el momento, entre artistas, personalidades políticas, colectivos de asociaciones, etc., donde tenemos finalizada 7 páginas hasta el momento, las cuales también han sido bordadas en distintas ciudades da España, así como en otras ciudades del mundo como en Otthensen - Hamburgo Alemania, Mukojima- Japón, Washington D. C.- EUA, gracias a la colaboración de muchas personas que apoyan la lucha de los vecinos del Cabanyal. Ha participado en el festival de arte urbano Intramurs en el estudio de la artista Silvia Molinero, así como realizamos diferentes convocatorias en distintos espacios públicos de la ciudad. Actualmente participa junto a diferentes colectivos en la Exposición Testigos de la Ciudad. Activismos Políticos y Culturales en la Comunidad Valenciana, Comisariada por Álvaro de los Ángeles con la coordinación con Sandra Moros, en el museo IVAM (Institut Valencià d'Art Modern). A través de un diario electrónico (WebBlog) se registra toda la acción.

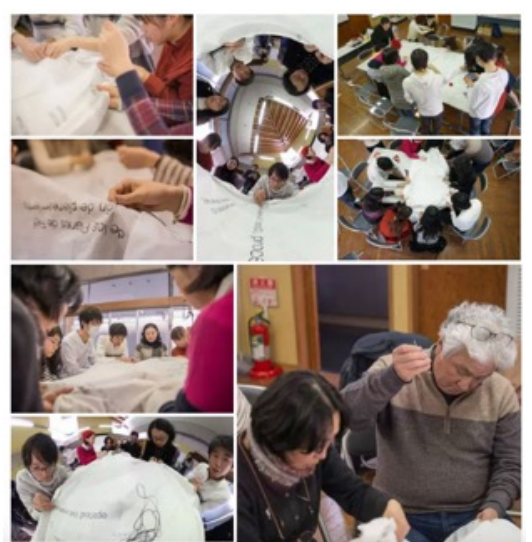

Bordando en Mukojima la página 5 de la orden MINISTERIAL CUL/3631/2009 DE 29 DE DICIEMBRE PARA SU CUMPLIMIENTO.

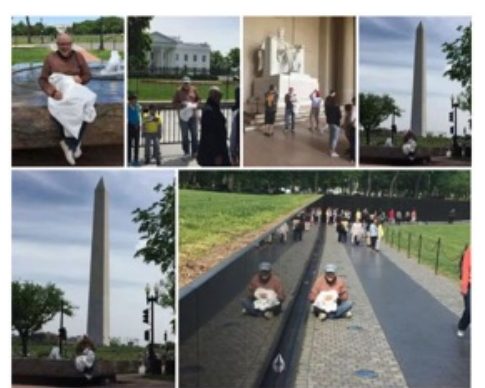

Bordando en Washinton D.C. la página 13 de la orden MINISTERIAL CUL/3631/2009 DE 29 DE DICIEMBRE PARA SU CUMPLIMIENTO.

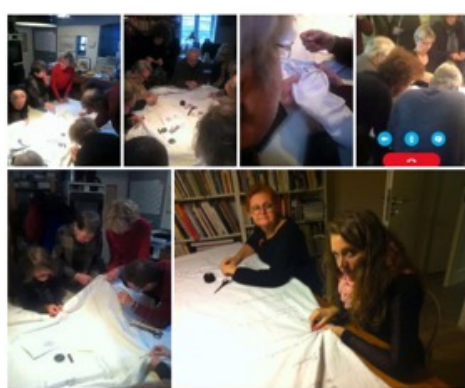

Bordando en Ottensen - Hamburgo la página 6 de la orden MINISTERIAL CUL/3631/2009 DE 29 DE DICIEMBRE PARA SU CUMPLIMIENTO.

Concluimos que la realización de una obra de arte colaborativa a partir de una acción de craftivismo, realizada en distintos ámbitos, a través de técnicas que normalmente se desarrolla en el ámbito doméstico, puede dialogar con el espacio público y al mismo tiempo ser una herramienta de protesta y reivindicación a través de proyectos colaborativos comprometidos socialmente, en los que la interacción y colaboración entre artista y espectador se ha establecido como un constante intercambio de experiencias. Desarrollar una obra colaborativa genera una cohesión social en la que todos comparten un mismo objetivo, y a la vez trabaja la autoestima de los participantes al convertirlos en protagonistas de sus propias causas y artífices de sus propias obras. Entre muchas de las conexiones que hay entre los proyectos presentados destacamos que todos ellos utilizan la Red como herramienta de ampliación y desdoblamiento de la intervención artística, muy importante hoy en día, ya que la Internet se ha convertido en un vehículo de mayor presencia dentro del mundo del arte, no solamente para realizar intervenciones artísticas, sino también como vehículo de difusión de las mismas, así como de generar redes de colaboraciones mutuas.

\footnotetext{
${ }^{4}$ CraftCabanyal, un proyecto de arte de craftivismo participativo con perspectiva de género, que empieza en 2013, coordinado por Bia Santos, donde procuramos hacer un diálogo entre el espacio privado con el espacio público a partir de las prácticas artesanales realizadas en general en el ámbito doméstico y vinculadas a la imagen de la mujer. Realizamos intervenciones artísticas dentro de un activismo político social, con la colaboración, artistas, vecinos y vecinas, amigos y simpatizantes del barrio, que tienen la oportunidad de expresar a partir del proceso creativo su visión sobre el barrio y a la vez participar de una obra artística en defensa del patrimonio material e inmaterial del barrio del Cabanyal en Valencia que estuvo 17 años amenazado por un proyecto de especulación urbanística. Se enmarca dentro de las diversas acciones realizadas por la Plataforma Salvem el Cabanyal. In: http://craftcabanyal.espai214.org/

${ }^{5}$ Colectivo ciudadano de defensa y concienciación ciudadana sobre esta situación y por extensión sobre la gestión de todo patrimonio histórico, que nace el 1998 en el barrio del Cabanyal en Valencia con el objetivo de evitar la prolongación de la avenida Blasco lbáñez la cual suponía derribar 1651 viviendas, proyecto que fue paralizado con el cambio de gobierno en 2015 y la respectiva derogación del Plan. http://www.cabanyal.com/

${ }^{6}$ Diario del proceso https://craftcabanyal.wordpress.com/
} 


\section{FUENTES REFERENCIALES.}

Bibliografía.

DUQUE, Félix. (2001). Arte Público y Espacio político. Madrid: Akal.

LIPPARD, Lucy R. (2001). Mirando alrededor: dónde estamos y dónde podríamos estar. Modos de hacer. Arte crítico, esfera pública y acción directa. Salamanca: Ediciones Universidad de Salamanca.

MARTíNEZ, E.; Dos Santos, F. (2014) Una herramienta de resistencia. Cabanyal Archivo Vivo: el archivo digital como estrategia de resistencia frente a las inercias urbanísticas de destrucción del patrimonio. En Actas del II Congreso Internacional de Educación Patrimonial. 28-31 Oct 2014. Madrid: IPCE/OEPE, pp. 689-702

MORRIS, W. (1977). Arte y Sociedad Industrial. Valencia: Fernando Torres Editor

SENNETT. R. (2013). Artesanía, Tecnología y Nuevas formas de Trabajo. Barcelona: Katz Editores.

SILVA DOS SANTOS, FC. (2015). Mujer, casa y nuevos medios: el arte de tejer en red, una propuesta experimental artística neomedial [Tesis doctoral no publicada]. Universitat Politècnica de València. doi:10.4995/Thesis/10251/58774, In: http://hdl.handle.net/10251/58774

RANCIÈRE, J. (2010). El espectador emancipado. Buenos Aires (Argentina): Manantial.

Electrónico.

BLANCO, P. (1989). Prácticas artísticas colaborativas en la España de los años noventa. , pp. 188-205. En: Desacuerdos. Sobre arte, políticas y esfera pública en el Estado español.

Disponible: http://ayp.unia.es/dmdocuments/des_c02.pdf [Consulta: 17/04/2014]

GARRIDO, A.P. (2009). El arte comunitario: origen y evolución de las prácticas artísticas colaborativas. Arteterapia. Papeles de arteterapia y educación artística para la inclusión social [en línea], vol. 4, pp. 197 - 211. ISSN 1988-8309. DOI -. Disponible en: http://revistas.ucm.es/index.php/ARTE/article/view/ARTE0909110197 A.[ Consulta: 04 de mayo de 2015]

CraftCabanyal http://craftcabanyal.espai214.org/ .[Consulta: 09/03/2017]

Cornelia Parker - Carta Magna - In: https://www.theguardian.com/artanddesign/jonathanjonesblog/2015/may/14/magna-carta-anembroidery-cornelia-parker-british-library-wikipedia-prisoners-jarvis-cocker .[Consulta: 15/03/2017]

Crochet Coral http://crochetcoralreef.org/satellite/chicago.php .[Consulta: 14/03/2017]

Plaraforma Salvem el Cabanyal http://www.cabanyal.com/ .[Consulta: 09/03/2017]

Tejiendo Calle - https://submarina.info/tejiendo-la-calle/.[Consulta: 09/03/2017]

Urban Knitting Barcelona http://urbanknittingbarcelona.blogspot.com.es/.[Consulta: 12/03/2017]

00 cabezas, 100 mujeres, 100 vendas moradas. In: http://uanflores.blogspot.com.es/ [Consulta: 12/03/2017]

Mujeres y Punto. Foto: Miguel Lorenzo. In: http://www.makma.net/tag/profesora-de-la-universitat-de-valencia/ [Consulta: 12/03/2017] 